\title{
Letter
}

\section{Wave propagation in the magnetized cores of white dwarf stars with ultra-relativistic degenerate electrons}

\author{
P. K. SHUKLA ${ }^{1}$, D. A. MENDIS ${ }^{2}$ \\ and S. I. K R A S H E N I N N I K O V 2 \\ ${ }^{1}$ International Centre for Advanced Studies in Physical Sciences, Institute for \\ Theoretical Physics, Faculty of Physics and Astronomy, Ruhr University Bochum, \\ 44780 Bochum, Germany \\ (ps@tp4.ruhr-uni-bochum.de) \\ ${ }^{2}$ Jacobs School of Engineering, University of California, San Diego, La Jolla, CA 92093, \\ USA
}

(Received 4 January 2011; accepted 4 February 2011;

first published online 10 May 2011)

\begin{abstract}
We discuss the dispersive properties of low-frequency electromagnetic (EM) perturbations in the magnetized core of self-gravitating white dwarf stars with ultra-relativistic degenerate electrons. For our purposes, we derive a dispersion relation by using the hydrodynamic equations for the ions under the action of EM and self-gravitating forces, and the inertialess electrons under the action of EM forces and the gradient of the ultra-relativistic pressure. The dispersion relation admits stability of a white dwarf star against a class of EM perturbations whose wavelengths are shorter than $15000 \mathrm{~km}$.
\end{abstract}

White dwarf stars [1], aptly named due to their very low luminosities yet high surface emissivities, are compact bodies with radii $\leqslant 10^{-2} R_{\odot}$ and masses typically $\leqslant M_{\odot}$. Consequently, their average bulk densities are quite high $\left(\sim 10^{30} \mathrm{~cm}^{-3}\right.$. At such high densities, the background electrons are degenerate and follow the Fermi-Dirac statistics since the inter-electron distance is of the order of the de Broglie thermal wavelength or even comparable with the Compton length. In such a situation, the electrons are crowded very close together and the combination of the Pauli exclusion principle (viz. two electrons cannot occupy the same energy state) and Heisenberg's uncertainty principle dictate that the position and momentum of of electrons cannot be precisely determined simultaneously, viz. the product of the uncertainties of the position and momentum $(\Delta x \Delta p)$ is greater than $\hbar / 2$, where $\hbar$ is the Planck constant divided by $2 \pi$. An electron has a continuous motion around the position it occupies. This motion exerts pressure on the surrounding medium, exactly as the thermal agitation of the molecules of a gas exerts its pressure. This pressure is called the pressure of electron degeneracy. The electron pressure $P$ (the momentum transfer per unit area), when electrons are ultra-relativistic, are given by Refs [2,3]

$$
P=\frac{1}{4}\left(3 \pi^{2}\right)^{1 / 3} \hbar c n_{e} \approx \frac{3}{4} \hbar c n_{e}^{4 / 3},
$$

where $c$ is the speed of light in vacuum. 
In his classic paper, Chandrasekhar [3] considered the gravitation equilibrium of dense stars with ultra-relativistic degenerate electrons, and demonstrated that the internal outward pressure that counterbalances the inward pull of its own gravitation is not the normal thermal pressure of a hot gas, such as in our own sun, but rather that provided by a degenerate relativistic electron gas. The ultra-relativistic electron pressure $P$, which is proportional to $n_{e}^{4 / 3}$, allowed Chandrasekhar to discover the critical Chandrasekhar limit, $M_{c}$, for the mass of such white dwarfs. This mass, which has since been called the Chandrasekhar mass, has been revised upwards from its empirical estimate of $0.91-1.44 M_{\odot}[4,5]$.

The most accurate mass determination of white dwarf stars, using binary orbit solutions, give values $\leqslant M_{\odot}$ (e.g. the mass of the most well-known white dwarf stars, Sirius B, the faint companion of the bright main sequence star, Sirius A, is estimated to be $\left(\sim 1 M_{\odot}\right)$, whereas its radius is estimated to be about $0.007 R_{\odot}$, e.g. see Ref. [6]. Incidentally, the above dimensions show that the average bulk density of Sirius $\mathrm{B} \approx 4.4 \times 10^{6} \mathrm{~g} \mathrm{~cm}^{-3}$. As the average electron number density is given by

$$
n_{e}=\frac{X}{m_{H}}+\frac{2 Y}{4 m_{H}}+\frac{Z}{\langle A\rangle m_{H}} \frac{\langle A\rangle}{2}=\frac{(1+X) \rho}{2 m_{H}},
$$

where $X, Y$ and $Z$ are the mass fractions of $H, H e$ and the heavy atoms of average atomic mass $\langle A\rangle$, and where it has been assumed that each of those constitute $\langle A\rangle / 2$ electrons, when fully ionized. Furthermore, $\rho$ is the mass density, while the estimated composition of the white dwarf stars may vary, as inferred from their spectra, many seem to be dominated by He. If, following Chandrasekhar [5], we assume that the composition is fully $\mathrm{He}[6,7]$, then $X=0$, and using (2) we obtain $n_{e} \approx 1.3 \times 10^{30} \mathrm{~cm}^{-3}$.

Following on his earlier work, Chandrasekhar [8] discussed the dynamical stability of white dwarfs, wherein he showed that, while general relativistic effects begin to play a role for the more massive white dwarf stars close to Chandrasekhar limit, this mass limit is decreased somewhat to $\sim 1.42 M_{\odot}$, due to the onset of dynamical instability for masses about this value [9].

In this paper, we confine ourselves to the stability of core of self-gravitating $[10,11]$ white dwarf stars $\left(M \leqslant M_{\odot}\right)$ with ultra-relativistic degenerate electrons and non-degenerate ions, but not subject to general relativistic effects discussed by Chandrasekhar [8]. We, however, take into account of the massive magnetic fields of such cores. There is a range of the magnetization of white dwarf stars, with the observed surface magnetic field ranging from as low as a few kilogauss to as high as 300 megagauss [6].

We model the core of the white dwarf star as a dense uniform, self-gravitating, magnetized electron-ion plasma, with ultra-relativistic degenerate electrons and nondegenerate ions. Here we do not deal with the global oscillations of the configuration, but rather with the dynamics of a novel class of waves, which are almost equally affected by electromagnetic (EM) and gravitational forces, resulting from density and magnetic field perturbations which may be self-excited or which may result from gravitational perturbations such as from a binary companion. We note that unlike the much smaller and much denser neutron stars, white dwarf stars are not known to be fast rotators. In fact, observations indicate that most white dwarfs rotate slowly, if at all [7]. So we do not need to consider the effect of rotation in our analysis. We do, however, consider the effects of magnetization, as discussed 
earlier. Of course, the morphology of the magnetic field within white dwarf cores is unknown. Therefore, in our present analysis, the magnetic field and the plasma number density are assumed to be uniform.

The dynamics of low-frequency (in comparison with the electron gyrofrequency), low phase velocity (in comparison with the speed of light in vacuum) electromagnetic disturbances in the core of a self-gravitating dense white dwarf electron-ion plasma is governed by the continuity equation:

$$
\frac{\partial n_{j}}{\partial t}+\nabla \cdot\left(n_{j} \mathbf{u}_{j}\right)=0
$$

the inertialess electron momentum equation:

$$
0=-n_{e} e\left(\mathbf{E}+\frac{1}{c} \mathbf{u}_{e} \times \mathbf{B}\right)-\frac{3}{4} \hbar c \nabla n_{e}^{4 / 3},
$$

the ion momentum equation:

$$
m_{i} n_{i} \frac{d \mathbf{u}_{i}}{d t}=n_{i} e\left(\mathbf{E}+\frac{1}{c} \mathbf{u}_{i} \times \mathbf{B}\right)-m_{i} n_{i} \nabla \phi_{g},
$$

Ampère's law

$$
\frac{\partial \mathbf{B}}{\partial t}=-c \nabla \times \mathbf{E}
$$

Faraday's law:

$$
\nabla \times \mathbf{B}=\frac{4 \pi \mathbf{J}}{c} \equiv \frac{4 \pi e}{c}\left(n_{i} \mathbf{u}_{i}-n_{e} \mathbf{u}_{e}\right)
$$

and Poisson's equation for the gravitational potential $\phi_{g}$ :

$$
\nabla^{2} \phi_{g}=4 \pi G n_{i} m_{i}
$$

together with the quasi-neutrality approximation $n_{e}=n_{i}=n$. Here we have denoted $d / d t=(\partial / \partial t)+\mathbf{u}_{i} \cdot \nabla$, and $J$ is the plasma current density.

In (1)-(8), we have denoted the number density of the plasma species $j$ ( $j$ equals $e$ for the electrons and $i$ for the ions) by $n_{j}$, the fluid velocity by $\mathbf{u}_{j}$, the electric and magnetic fields by $\mathbf{E}$ and $\mathbf{B}$, respectively. Also, $e$ is the magnitude of the electron charge, $m_{i}$ is the ion mass and $G$ is the gravitational constant. The electron inertial force in (4) has been neglected by assuming that $\partial^{2} n_{1} / \partial t^{2} \ll \lambda_{C} n_{0}^{1 / 3} c^{2} \nabla^{2} n_{1}$, where $n_{1}=\left(n-n_{0}\right) \ll n_{0}, n_{0}$ is the unperturbed electron number density, $\lambda_{C}\left(=\hbar / m_{e} c\right)$ is the Compton length and $m_{e}$ is the electron mass.

By using (4) we can eliminate the electric field from (5) obtaining

$$
m_{i} n \frac{d \mathbf{u}_{i}}{d t}=\frac{(\nabla \times \mathbf{B}) \times \mathbf{B}}{4 \pi}-\frac{3}{4} \hbar c \nabla n^{4 / 3}-m_{i} n \nabla \phi_{g}
$$

where we have used (7) to eliminate J. Furthermore, the combination of (5) and (6) yields

$$
\frac{\partial \mathbf{B}}{\partial t}=\nabla \times\left(\mathbf{u}_{i} \times \mathbf{B}\right)-\frac{c m_{i}}{e} \nabla \times\left(\frac{d \mathbf{u}_{i}}{d t}\right) .
$$

Equations (8)-(10) and

$$
\frac{\partial n}{\partial t}+\nabla \cdot\left(n \mathbf{u}_{i}\right)=0
$$


are the governing nonlinear 'gravito-magnetohydrodynamic (G-MHD)' equations for the low-frequency (in comparison with the electron gyrofrequency) EM waves in a self-gravitating dense plasma with ultra-relativistic degenerate electrons. Our G-MHD equations can be used for investigating the properties of linear EM waves and nonlinear EM wave phenomena (three-dimensional EM wave turbulence and associated turbulent spectra, formation of EM wave structures, etc.)

In the following, we consider the linear propagation of low-frequency (in comparison with the ion gyrofrequency) EM waves with the density perturbation $n_{1}$ and the compressional magnetic field $B_{z}\left(\ll B_{0}\right)$, where $B_{0}$ is the strength of the homogeneous magnetic field that is aligned along the $z$-axis in a Cartesian coordinate system. Accordingly, we linearize (8)-(11) by supposing that $n=n_{0}+n_{1}$ and $\mathbf{B}=\left(B_{0}+B_{z}\right) \hat{\mathbf{z}}$, where $\hat{\mathbf{z}}$ is the unit vector along the $z$-axis, and combine the resultant equation to obtain for $\omega \ll \omega_{c i}$ :

$$
\left(\frac{\partial^{2}}{\partial t^{2}}-C_{\hbar A}^{2} \nabla^{2}\right) \nabla^{2} n_{1}-\Omega_{J}^{2} \nabla_{\perp}^{2} n_{1}=0,
$$

where we have neglected the sheared magnetic field perturbation and the ion dynamics parallel to $\hat{\mathbf{z}}$, and used the frozen-in-field relation $B_{z}=B_{0} n_{1} / n_{0}$. We have denoted $\omega_{c i}=e B_{0} / m_{i} c, \Omega_{J}=\left(4 \pi G n_{0} m_{i}\right)^{1 / 2}$ and $C_{\hbar A}=\left(C_{A}^{2}+C_{\hbar}^{2}\right)^{1 / 2}$, where $C_{A}=B_{0} /\left(4 \pi n_{0} m_{i}\right)^{1 / 2}$ is the Alfvén speed and $C_{\hbar}=c\left(\hbar n_{0}^{1 / 3} / m_{i} c\right)^{1 / 2}$ is the ion oscillation frequency associated with the combined action of the ion inertial force and the ultra-relativistic degenerate electron pressure gradient.

Assuming that $n_{1}$ is proportional to $\exp (-i \omega t+i \mathbf{k} \cdot \mathbf{r})$, where $\omega$ and $\mathbf{k}$ are the frequency and the wave number, respectively, from Fourier transform (12) to obtain

$$
\omega=\left(k_{\perp}^{2} C_{\hbar A}^{2}-\Omega_{J}^{2} \frac{k_{\perp}^{2}}{k^{2}}\right)^{1 / 2},
$$

where $k=\left(k_{\perp}^{2}+k_{z}^{2}\right)^{1 / 2}$ and $k_{\perp}\left(k_{z}\right)$ is the component of the wave vector $\mathbf{k}$ across (along) the external magnetic field direction. Equation (13) admits instability if the wavelength of the obliquely (against $\hat{\mathbf{z}}$ ) propagating EM perturbation

$$
\lambda>2 \pi C_{\hbar A} / \Omega_{J},
$$

where $\lambda=2 \pi / k$.

In order to have some appreciation of our result, we take some typical parameters that are representative of the core of a white dwarf star, and estimate the wavelength of the EM perturbations in our instability, viz. $n_{0}=1.3 \times 10^{30} \mathrm{~cm}^{-3}, B_{0}=3 \times 10^{8} \mathrm{G}$. By using the physical constants $\hbar=1.05 \times 10^{-27} \mathrm{erg} \mathrm{s}^{-1}, G=6.67 \times 10^{-8}$ dyn $\mathrm{cm}^{-2} \mathrm{~g}^{-2}, m_{i}=4 \times 1.67 \times 10^{-24} \mathrm{~g}$ and $c=3 \times 10^{10} \mathrm{~cm} \mathrm{~s}^{-1}$, we obtain $\Omega_{J} \sim 2 \mathrm{~s}^{-1}$, $C_{A}=1.1 \times 10^{5} \mathrm{~cm} \mathrm{~s}^{-1}$ and $C_{\hbar} \approx 5 \times 10^{8} \mathrm{~cm} \mathrm{~s}^{-1}$. It then turns out that a selfgravitating white dwarf star will remain stable against EM perturbations, since the wavelength of the EM perturbations, deduced from (14) turns out to be of the order of $1.57 \times 10^{4} \mathrm{Km}$, which is larger than the radius of a white dwarf core.

To summarize, we have considered the propagation of the low-frequency EM waves in a self-gravitating magnetized plasma in the core of white dwarf stars in which ultra-relativistic electrons are degenerate. Accounting for the EM and selfgravitating forces, and using the ultra-relativistic pressure law for the degenerate electrons, we have derived a set of nonlinear equations for EM by using the two fluid equations, the Poisson equation for the gravitational potential, Ampère's law 
and Faraday's law. In the linear regime, we have derived a wave equation which yields a dispersion relation. The latter admits stability of a white dwarf star because the wavelength of the EM perturbations in our G-MHD system is larger than the radius of the star for typical plasma parameters that are representative of the core of a dense white dwarf star. In the present analysis, we have confined ourselves to the core of a white dwarf star where, it can be shown, using a 'polytrope' model, that more than $90 \%$ of the mass resides. For mathematical simplicity, we have assumed this core to be of uniform density and carry a uniform magnetic field. In reality, the density throughout a white dwarf star varies with radius. This leads to the electrons to be relativistically degenerate in the core, while being non-relativistically degenerate in an outer mantle and non-degenerate in a thin outer atmosphere. There would be corresponding variations of the magnetic field as well. We propose to address, this admittedly, more involved, problem in a future paper. Yet, even with this analysis of a simplified model, we have been able to obtain interesting insights into the propagation of low-frequency (in comparison with the ion gyrofrequency) EM waves within the core of such stars.

\section{Acknowledgments}

This research was partially supported by the Deutsche Forschungsgemeinschaft (DFG) through the project SH21/3-1 of the Research Unit 1048 at RUB, and by the US Department of Energy (DOE) through the grant DE-FG02-04ER54852 at UCSD.

\section{References}

[1] Shapiro, S. L. and Teukolsky, S. L. 1983 Black Holes, White Dwarfs, and Neutron Stars: The Physics of Compact Objects. New York: John Wiley \& Sons.

[2] Chandrasekhar, S. 1931 Phil. Mag. 11, 591.

[3] Chandrasekhar, S. 1931 Astrophys. J. 74, 81.

[4] Chandrasekhar, S. 1935 Mon. Not. R. Astron. Soc. 170, 405.

[5] Chandrasekhar, S. 1939 An Introduction to the Study of Stellar Structure. Dover Publications, pp. 412.

[6] Libert, J. 1980 Ann. Rev. Astron. Astrophys. 18, 363.

[7] Gatewood, G. D. and Gatewood, C. V. 1978 Astrophys. J. 225, 191.

[8] Chandrasekhar, S. 1964 Phys. Rev. Lett. 12, 114.

[9] Chandrasekhar, S. and Tooper, R. F. 1964 Astrophys. J. 139, 1396.

[10] Avinash, K. and Shukla, P. K. 1994 Phys. Lett. A 189, 470.

[11] Shukla, P. K. and Stenflo, L. 2006 Phys. Lett. A 355, 378. 